$10-1-2008$

\title{
Future Implementation of Adult Stem Cell Therapy in the Current Standards of Care for Myocardial Infarction
}

Paul D. Bates

Nova Southeastern University

Follow this and additional works at: https://nsuworks.nova.edu/ijahsp

Part of the Medicine and Health Sciences Commons

\section{Recommended Citation}

Bates PD. Future Implementation of Adult Stem Cell Therapy in the Current Standards of Care for Myocardial Infarction. The Internet Journal of Allied Health Sciences and Practice. 2008 Oct 01;6(4), Article 11.

This Manuscript is brought to you for free and open access by the College of Health Care Sciences at NSUWorks. It has been accepted for inclusion in Internet Journal of Allied Health Sciences and Practice by an authorized editor of NSUWorks. For more information, please contact nsuworks@nova.edu. 


\title{
Future Implementation of Adult Stem Cell Therapy in the Current Standards of Care for Myocardial Infarction
}

\begin{abstract}
Purpose: Adult stem cells are among the new methods of approaching the treatment of myocardial tissue damage. The purpose of this review is to clarify misconceptions about stem cell therapy efficacy in clinical trials and provide a thorough understanding of adult stem cells as a future treatment for patients with myocardial infarction. Methods: A comprehensive review of literature was performed analyzing and comparing 12 clinical trials involving the treatment of patients with acute and chronic myocardial infarction. Results: Stem cell treatments carry an excellent safety profile with the ease of one-time dosing, and have shown dramatic functional improvements while reducing the recurrence of myocardial infarction and enhancing quality of life. Important changes with adult stem cell treatments include 1) formation of new cardiomyocytes, 2) sufficient and sustained improvements in cardiac output, 3) increased myocardial contractility, 4) decreased infarct zone diameter, 5) increased left ventricular function, 6) increased exercise ability, and 7) increased coronary perfusion secondary to neovascularization. Conclusion: At this time, based on the current clinical evidence, adult stem cell therapy is in a position to be considered as an optional treatment for patients with acute or chronic myocardial infarction. Adult stem cell therapy is still in experimental stages of development and the continued clinical involvement will provide more evidence to the therapeutic effects of the treatment.
\end{abstract}




\title{
IIAAHSP \\ The Internet Jourfal of Allied Health Sciences and Practice \\ http://ijahsp.nova.edu
}

A Peer Reviewed Publication of the College of Allied Health \& Nursing at Nova Southeastern University

Dedicated to allied health professional practice and education

http://ijahsp.nova.edu Vol. 6 No. 4 ISSN 1540-580X

\section{Future Implementation of Adult Stem Cell Therapy in the Current Standards of Care for Myocardial Infarction}

\author{
Paul D. Bates, 1st Lt. USAF, MMS, PA-C \\ United States Air Force, (Written while student at Nova Southeastern University Physician Assistant Program, Ft. Lauderdale, FL.) \\ United States
}

CITATION: Bates, PD. Future Implementation of Adult Stem Cell Therapy in the Current Standards of Care for Myocardial Infarction. The Internet Journal of Allied Health Sciences and Practice. Oct 2008, Volume 6 Number 4.

\begin{abstract}
Purpose: Adult stem cells are among the new methods of approaching the treatment of myocardial tissue damage. The purpose of this review is to clarify misconceptions about stem cell therapy efficacy in clinical trials and provide a thorough understanding of adult stem cells as a future treatment for patients with myocardial infarction. Methods: A comprehensive review of literature was performed analyzing and comparing 12 clinical trials involving the treatment of patients with acute and chronic myocardial infarction. Results: Stem cell treatments carry an excellent safety profile with the ease of one-time dosing, and have shown dramatic functional improvements while reducing the recurrence of myocardial infarction and enhancing quality of life. Important changes with adult stem cell treatments include 1) formation of new cardiomyocytes, 2) sufficient and sustained improvements in cardiac output, 3) increased myocardial contractility, 4) decreased infarct zone diameter, 5) increased left ventricular function, 6) increased exercise ability, and 7) increased coronary perfusion secondary to neovascularization. Conclusion: At this time, based on the current clinical evidence, adult stem cell therapy is in a position to be considered as an optional treatment for patients with acute or chronic myocardial infarction. Adult stem cell therapy is still in experimental stages of development and the continued clinical involvement will provide more evidence to the therapeutic effects of the treatment.
\end{abstract}

\section{INTRODUCTION}

Acute and chronic myocardial infarctions (MI) represent significant causes of morbidity and mortality throughout the world today. According to the CDC National Vital Statistics Report released in January 2008, heart disease remains the leading cause of death for adults in the United States. ${ }^{1}$ A variety of factors are recognized that may contribute to an increased risk of myocardial ischemia and infarction, including poor diet, smoking, environmental causes, genetic predisposition, coronary artery disease, diabetes or trauma. Considering the difficulty in controlling all predisposing factors, treatment for post-injury is particularly important.

During myocardial ischemia, deprivation of oxygen to the tissue is detrimental to cardiac function. The cardiomyocytes that are unaffected by the injury are required to compensate for the loss in function, thus leading to ventricular dilation and wall thinning. This causes an increased stress on the ventricle which ultimately leads to hypertrophy of the cardiomyocytes. The most problematic effect of ventricular dilation is heart failure, resulting in a decreased cardiac output and function, and increasing the morbidity and mortality among these patients.,3 Moreover, because of the limited regenerating capability of myocardial tissue, there is a reduction in the chance that full recovery will be a practical goal after MI.

Currently, there are various medical interventions available such as medications, catheterization, and stent placement that may facilitate a return in cardiac function after myocardial damage, but there is often a limited amount of improvement. The initial goal in treatment of acute $\mathrm{MI}$ is reperfusion with percutaneous intravenous intervention $(\mathrm{PCl})$ and ablation of clots with thrombolytic

(c) The Internet Journal of Allied Health Sciences and Practice, 2008 
therapy. ${ }^{3}$ Treatment of $\mathrm{Ml}$ is currently focused on reducing the extent of ischemic damage and increasing the non-infarcted tissue function to replace damaged tissue. Since myocardial repair is limited with the use of current treatment techniques, new options are needed for more effective cardiac revival, whether as a novel treatment or a supplement to current therapy.

Research is continuing throughout the world on the use of adult stem cells to treat many diseases and injuries. New studies have provided a variety of ways to treat both acute and chronic MI utilizing stem cells that can be found in various regions throughout the adult human body. Even though there has been extensive research done, standard treatments with stem cells have not been accepted because of many factors, including lack of understanding and large population testing.

Stem cells, by nature, are the primitive form of all specialized cells in the body. Through successive divisions, the cell is capable of producing any specialized cell in the body, including cardiac muscle. It is a misconception that all stem cells are derived from a fetus or embryo. Though a fetus can provide cells in their primitive form, stem cells may also be obtained from various parts of the adult body; they simply have less potential to differentiate without assistance. The major sources of adult stem cells used for treatment of Ml include bone marrow, healthy cardiac tissue, circulating blood, and skeletal muscle tissue. ${ }^{4-7}$ Cells can be collected in their undifferentiated state from these areas, cultured in media with growth factors and other chemicals, and replaced into the body to treat injury. Embryonic, umbilical and, fetal, are all potential sources of stem cells, however these sources are unrelated to the patient they are intended to treat; thus, embryonic, umbilical and fetal stem cells carry risks of rejection in the treated individual. In the case of adult stem cells, they can be obtained directly from the patient being treated, thus eliminating the potential risk for rejection. Research has shown that adult stem cells are effective in the treatment of myocardial injury by regenerating cardiac muscle tissue as well as inducing the formation of new blood vessels in the region of infarction. ${ }^{5}$ Progressing from basic tissue research to animal trials, along with human studies outside the United States, the potential for the use of stem cells as a treatment for Ml is promising.

The purpose of this review is to clarify misconceptions about stem cell therapy efficacy in clinical trials and provide a thorough understanding of stem cells as a future treatment for patients with MI. Compiled data from recent clinical studies show that adult stem cells are at a stage in which they can be used in combination with current standard of treatment to provide an optimal recovery of cardiac function in acute or chronic Ml patients. As a result of the different studies and experiments, it has been determined that there is very little risk and few side effects for adult stem cell use in the treatment of acute and chronic MI. In contrast, many current treatments carry a large assortment of side effects and the efficacy varies between patients. Some current interventions hold the potential to be more life threatening to the patient than the infarct itself. The use of adult stem cells has shown to be a beneficial way to treat patients who experience myocardial infarction, and consideration should be given to the adoption of standard treatment to reverse the detrimental outcomes from ischemic damage to the myocardium.

\section{METHODS OF RESEARCH}

As a review of the literature, this study did not involve live subjects or interviews. Inclusion criteria for the present study include articles published after January 2000 in English or an English translation from a wide variety of journals, with subject heading acute and/or chronic myocardial infarction and adult stem cell treatment and/or transplantation. Search methods included a variety of resources provided by Nova Southeastern University in the form of original printed journals or online accessible journals. Though human trials were the focus of research, animal trails were also reviewed and considered a vital portion of this review. Articles were not included in data presentation if it was not an original study, included treatment of heart failure not secondary to $\mathrm{Ml}$, or if the study exclusively involved granulocyte colony-stimulating factor in the trial. Those studies that discussed embryonic stem cells were completely omitted from this review. There were 12 clinical studies analyzed for the presentation of clinical evidence in adult stem cell therapy.

\section{CURRENT STANDARDS OF CARE}

According to an expert consensus document on the definition of myocardial infarction, the term should be used whenever there is an indication of necrosis. ${ }^{8}$ Table 1 summarizes the clinical criteria given by Thygesen and associates for the identification of acute and chronic Ml. When a patient is diagnosed with acute Ml, prompt treatment is the most important deciding factor in the extent of the cardiac injury. The current treatment for both acute and chronic Ml involves similar methods attempting to limit ischemic damage and recover as much of the viable myocardium as possible.

The current ACC/AHA Guidelines recommend, in the incidence of acute MI, that the best results for patient recovery are immediate PCl within 90 minutes of initiation of medical care..$^{9,10} \mathrm{In}$ the acute care of $\mathrm{Ml}$, it is also recommended that a variety of medications are utilized to reduce the amount of ischemia by recovering coronary blood flow. The medications include nitrates, analgesics, beta-adrenergic blockers, calcium channel blockers, angiotensin-converting enzyme inhibitors (ACE-I), antiplatelet/ anticoagulation therapy, and fibrinolytic therapy. After the acute treatment period, patients often remain on chronic therapy to 
improve long-term prognosis with beta-blockers, calcium channel blockers, anti-lipid medications, ACE-I with or without the addition of a diuretic, and/or antiplatelet therapy. $., 9,10$ The current treatment for myocardial infarction is aimed at providing the best recovery for patients and preventing complications of heart failure, but new therapies are warranted to further improve patient outcomes.

Table 1. Criteria for Acute and Chronic Myocardial Infarction ${ }^{\mathrm{a}}$

\begin{tabular}{|c|c|c|}
\hline \multicolumn{2}{|r|}{ Acute Myocardial Infarction } & Chronic/Prior Myocardial Infarction \\
\hline & 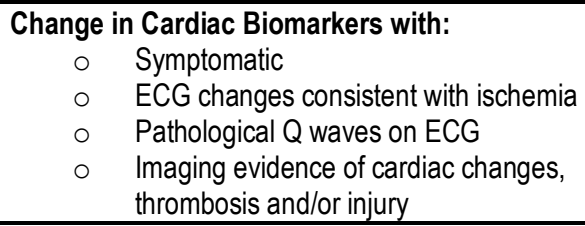 & $\begin{array}{ll}\text { - } & \text { New pathological Q wave with/ without symptoms } \\
\text { - } & \text { Imaging evidence of myocardial changes and injury } \\
\text { - } & \text { Pathological findings of myocardial injury }\end{array}$ \\
\hline$\bullet$ & Cardiac death & \\
\hline$\bullet$ & Infarction associated with $\mathrm{PCl}$ & \\
\hline & Infarction associated with CABG & \\
\hline$\bullet$ & Infarction associated with stent thrombosis & \\
\hline
\end{tabular}

The goal of stem cell therapy is to provide an adjunct to the current post-Ml treatment modality. It would not replace the current standards of care, but add an additional option to optimize the recovery of myocardial tissue after infarction. In clinical studies of adult stem cell therapy for MI, stem cell treatment patients did not receive any different post-treatment than the control patients, and all patients remained on the typical regimen of post-infarction medications after hospitalization. 6,11 With the adjunct of adult stem cell therapy as a low-risk optional treatment, practitioners may find this new modality the ideal choice to salvage damaged myocardium.

\section{DIFFERENTIATION AND AVAILABILITY OF ADULT STEM CELLS Stem Cell Differentiation}

The mature heart is naturally unable to completely regenerate itself with new cardiac muscle cells, or cardiomyocytes. Cardiac muscles cells are highly specialized and are not easily replenished; instead, they are replaced by nonfunctional scar tissue. Scar tissue is incapable of performing the vital functions of cardiac muscle, and the heart often suffers decreases in compliance and cardiac output. Stem cells are the fundamental root of all cells and are capable of differentiating into any type of cell through successive divisions, whereas other cells are limited in their ability to become specialized tissue, such as cardiac or neural. Since the heart is comprised of very specialized cardiac muscle cells, stem cells are important in the regeneration of this tissue. The basic level of stem cell research is determining cell capability and growth requirements. In an effort to determine whether it is possible to generate cardiomyocytes from stem cells, several studies have been done with tissue and cell samples. These studies have established that cardiomyocytes can be created in the laboratory setting and hold the potential to be used in cell transplantation. ${ }^{12,13}$

In an analysis of deceased human hearts that suffered from hypertension or coronary heart disease, it was determined that the heart contains cells in their primitive state, which hold the potential to differentiate into new myocytes. Stem cell markers used to identify proteins in the cells found new cell formation in the hypertrophied areas of the heart. ${ }^{14}$ This finding was important because cardiac cells were commonly thought to be irreplaceable and the typical increases in heart mass were a result of hypertrophy of only existing cells. However, it was found that the heart not only replaces some of the cardiomyocytes, but that it possesses a reserve of stem cells. ${ }^{14}$

In a similar study, male heart transplant recipients that were given female hearts were biopsied and found to have new cardiomyocytes of non-cardiac origin within the foreign heart. ${ }^{15}$ Genetic changes were observed by detecting the male $Y$ chromosome within the cardiomyocyte nuclei. As a control, non-transplanted female myocardial tissue biopsies were analyzed and lacked the presence of $Y$ chromosomes. In addition, a male non-transplanted heart was analyzed and found to carry the $Y$ chromosome in approximately $66.47 \%$ of the cardiomyocytes. When examining the male recipient's new heart several months after transplant, the presence of $Y$-chromosomes (less than $0.2 \%$ ) indicated that new cells were formed. It is important to consider that female hearts do not contain the $Y$ chromosome, and since only $66 \%$ of the male heart contains $Y$ chromosomes, it is probable that a greater amount of regeneration occurred than detected in the study. Unfortunately, the heart is not capable of 
producing enough new cardiomyocytes to recover from the tissue loss from a typical Ml. As a result, it was suggested that, "although the percentage of regenerated cardiomyocytes is rather low, the mechanism could be used to replace damaged myocardium by raising the numbers and by promoting the migration of the respective precursor cells into the damaged myocardium."15

The most intriguing tissue experimentations involve the growth of beating cardiomyocytes in the laboratory. When cultured under specific conditions, bone marrow stem cells (BMSCs) can be differentiated into cardiac muscle cells that beat on their own after a two-week period, and synchronously in three weeks. It was also found that the cells formed into specific types of cardiac muscle cells (sinus node and ventricular) based on their electrical action potentials..$^{12}$ In a study by Matsuura and associates, Sca- $1^{+}$cells (cardiac stem cells) were acquired from the hearts of wild mice and used to grow cardiomyocytes in the lab..$^{13}$ They were able to culture the cells and develop cardiac cells that would beat in the media. The differentiated Sca- $1^{+}$cells were then tested for other cardiac properties by exposing them to various cardiac drugs, including Isoproterenol, which has the ability to increase heart rate. The addition of cardiac drugs established that the cultured cells had the rate properties of typical cardiomyocytes by increasing and decreasing the rate of beating.

An important factor in basic stem cell research is determining if the stem cells utilized in therapy are actually incorporated into the heart. Stem cells have not only shown the ability to differentiate into new myocardial tissue, but that differentiation only occurs in the presence of ischemia or other chemical signals. ${ }^{16}$ The cell engraftment exclusively in damaged myocardium indicates that, in fact, it is the stem cell infusion that is causing myocardial remodeling in patients receiving the treatment. Since basic tissue studies have provided fundamental evidence that stem cells can be induced to differentiate into beating cardiomyocytes, this shows the potential for adult stem cells to be used in the treatment of cardiac tissue injury.

\section{Availability of Adult Stem Cells}

Red bone marrow is the most common, and possibly the most efficient, site for stem cell collection. Bone marrow is a major manufacturing center for cells and a storage facility for a variety of stem cells. The cells contained in the bone marrow vary in the degree of differentiation, but can be separated using centrifugation or other available methods of cell separation. To maximize results, the bone marrow stem cells (BMSCs) are aspirated from the large bones, most commonly the iliac crest, then mixed with heparin and filtered. 4,17,18 Large quantities of the BMSCs can be collected or the cells may be grown from a small quantity in growth media, and can then be delivered to the patient for treatment.16,19,20 In some cases, specific types of BMSCs have been isolated and used for treatment of MI, such as mesenchymal stem cells (MSCs), which were found in collected bone marrow to successfully treat rat models with cardiac injury. The MSCs are a rare type of cell found in bone marrow, but are descendants of the original stem cell, making them more differentiated. When used to treat cardiac ischemia they have been found to be highly effective through their ability to become cardiomyocytes.,17,21

In addition to bone marrow, other less common sources of stem cells that have the potential to become cardiomyocytes include the heart tissue itself, circulating blood, and skeletal muscle. It has been found that the heart contains cells in the primitive state, which hold the potential to be used for treatment of cardiac injury. ${ }^{13}$ Since the heart contains the well-differentiated myocytes that do not have the ability to repair damaged tissue, the stem cells have to be isolated from the tissue collection. Cardiac stem cells isolated and cloned from the heart ventricles of rat subjects have shown to be effective in the treatment of myocardial ischemia in the rat model, therefore making the heart a viable source of stem cells for myocardial repair. ${ }^{5}$ Circulating progenitor cells (CPCs) are an additional cell type easily obtained though venous blood centrifugation. After collection of a sufficient amount of CPCs and injection into the region of damage, the treatment successfully increases cardiac perfusion and function. ${ }^{6}$ However, CPCs were shown to be less effective than BMSCs in treating patients with chronic Ml. ${ }^{22}$ In several clinical trials, granulocyte colonystimulating factor (G-CSF) was used to mobilize the stem cells from the bone marrow into circulation. The target of G-CSF administration is to increase the CPC concentration in blood to allow for sufficient collection. In addition, G-CSF will theoretically influence an increase in the incidence of natural regeneration secondary to the larger cell population in circulation. In these studies that utilized G-CSF, the CPCs were found to be more effective at regenerating myocardium than when used alone, therefore making them a viable source of stem cells for MI treatment.23,24 In addition, skeletal myoblasts have also been identified as useful precursors to cardiac muscle tissue. ${ }^{7}$ Although skeletal myoblasts have not been tested in humans, these cells possess the ability to function as cardiac tissue when collected from the hind leg muscles of rats, cultured in growth medium and used to treat Ml.

Bone marrow stem cells, cardiac stem cells, blood-derived stem cells and skeletal myoblasts have all been identified as effective types of cells in treatment of cardiac injuries. However, further studies are warranted to establish which type of stem cell is most effective and ideal for treatment of Ml. It is possible that combinations or larger amounts of the different stem cells could be used to more effectively treat MI. 


\section{ADMINISTRATION OF STEM CELLS}

During administration of adult stem cells to treat myocardial infarction, the goal is to deliver enough cells to optimize repair in a manner that creates the least amount of side effects. The objective of cell isolation is to collect a sufficient number of cells for delivery. After collection, the cells are immediately isolated and either suspended or allowed to cultivate overnight. Following cultivation and suspension of the cells, it is important that the cells are administered in a heparin solution to prevent thromboembolus formation. Stem cell therapy is currently studied in patients that have already received the PCl treatment with or without stent placement. The ideal period of time to deliver the cells after primary catheterization has not been fully identified, but clinical evidence indicates that waiting more than 4 days has more benefit than immediate treatment. ${ }^{25,26} \mathrm{In}$ addition, the most successful method of delivering the cultivated stem cells has not been completely established, but can include transvascular cardiac catheterization, direct needle injection, and peripheral intravenous (IV) administration.

The most commonly utilized method of stem cell delivery is the transvascular approach by over-the-wire balloon catheterization of the infarcted coronary artery and injection of the cells directly into the infarct region. Injection with this method optimizes the repair of the infarcted region because the cells are introduced directly to the targeted tissue. ${ }^{17}$ The majority of reported stem cell therapy complications, however, occur from balloon catheterization and include local dissection of the artery and local wound infection at the site of catheter insertion. ${ }^{22}$ All clinical studies reported in this review utilized balloon catheterization when the stem cells were administered to the study patients.

In animal models, direct needle injection of the stem cells into the infarcted regions of the heart has been used. 4,7 This direct injection is an effective method of delivery for stem cells; however, it may not be ideal for human patients because of the need for an open-chest procedure. The opportunity to utilize this method of delivery is during an open-heart surgical procedure, such as a bypass, where the heart is already visualized and the surgeon can inject the stem cells into the regions of infarction. None of the clinical studies analyzed in this review utilized this method of administration. Direct injection of stem cells during transplant is a theoretical technique currently only used in laboratory research.

Peripheral IV administration of drugs is very common in the treatment of Ml as well as other injuries. In administration of stem cells via peripheral IV, concerns arise over the capability of the cells to repair the regions of infarction. Since the cells are administered through a peripheral vein, there is uncertain whether the cells will travel and engraft in the damaged area of the heart. In a study performed on rat subjects, it was found that IV administration is effective in reducing the infarct size and regenerating the myocardial tissue compared to control subjects. In addition, the stem cells were found to have induced new blood vessel formation in the ischemic tissue, thus allowing for increased blood perfusion to the damaged region. ${ }^{27}$ Though neovascularization has occurred in studies with central transcatheterization, the use of IV therapy could be more effective considering the cells have the advantage of reaching the tissue and vessels surrounding the infarct region. When using direct infusion, the cells primarily reach the area they are injected into, whereas IV administration is not necessarily limited to the immediate infarcted region. It is possible that the stem cells will also repair areas of the heart damaged during any previous injury and not detected by imaging, therefore preventing any future problems in that region. Since IV administration is safer for use on humans than catheterization, clinical trials utilizing IV therapy with humans would be a critical step for the standardization of stem cell treatment.

\section{STEM CELL DETECTION}

During the course of patient treatment, a variety of testing methods have been used to determine the viability of the stem cells and improvement to the myocardium. Echocardiography and angiography are the most common tests done to determine the patient's cardiac function and output. The common cardiac volumes calculated with an echocardiogram include the end-diastolic volume (EDV), end-systolic volume (ESV) and stroke volume. Left ventricular ejection fraction (LVEF) delineates the volumes determined by the echocardiogram. Since the LVEF is the percentage (fraction) of blood ejected from the left ventricle with each contraction, end-diastolic and stroke volumes are important. Though LEVF may vary depending on patient size, in a healthy individual a LVEF over $55 \%$ is ideal, whereas in many cases of acute myocardial injury, the ejection fraction can drop below $50 \%$. When the calculated ejection fraction falls under $25 \%$, there is indication of significant heart failure and can represent critical damage to cardiac muscle.

Aside from calculated cardiac improvement, other methods to detect stem cell transplantation include the use of primers, magnetic resonance imaging (MRI), single photon emission computed tomography (SPECT), and position emission tomography (PET). These methods vary in their sensitivity of stem cell detection but are regularly utilized. All are able to provide similar or more accurate readings of volumes and LVEF compared to angiography or echo, but they are too expensive to be used in standard assessment of cardiac improvement. The additional methods of imaging are most useful in clinical studies because they provide more sensitive information with visualization of the infarct region and the amount of improvement from therapy. In 
an animal study reported by Liu and associates, primers were used on the transplanted stem cells to track their implantation into the heart. They were able to show that the stem cells differentiated into cardiomyocytes by tracking the primer in pathological analysis of the myocardium after treatment. ${ }^{16}$ In the TOPCARE AMI trial, echocardiography was used in initial calculations of cardiac improvement and MRI was used around 9 days and 4 months post-therapy to corroborate the original observations.6,28 The ability of MRI studies to provide a 3-dimensional image proves the study more reliable than echo alone. Other clinical studies have employed multiple methods of imaging with MRI, and SPECT or PET scanning, which are all conclusive in determining that stem cells are effective in regenerating myocardium and offer similar data in the myocardial changes. ${ }^{23,29,30}$

\section{CLINICAL EVIDENCE}

Clinical trials involving treatment of patients with Ml using adult stem cells were first published in 2002, after animal experiments showed strong evidence that the therapy was effective in repairing damaged myocardium. Important changes in LVEF, coronary perfusion, myocardial contractility, infarct size, and exercise ability are the most effective methods of indicating the efficacy of cardiac treatment. The analysis of 12 clinical trials has provided evidence of beneficial ventricular remodeling and myocardial recovery from ischemia, and furthermore, established that adult stem cells are a reasonable treatment for MI. 6,11,17,22-26,29-32 All studies thus far have utilized either BMSCs or CPCs in random controlled environments administered via over-the-wire balloon catheterization after initial PCI. Patients in the cell treatment groups received the same standard of care therapy as the control group in addition to the stem cell treatment. A majority of the studies consisted of a small study group of approximately 30 to 60 patients divided between control and treatment groups. Two of the studies had patient groups greater than 100, but there has not been a clinical study with an enrollment larger than 200 patients.

The treatment of MI with adult stem cells has clear clinical benefits for patients. Increased cardiac output is the most commonly reported advantage of stem cell therapy. As presented in Figure 1, it is evident that the change in ejection fraction in the stem cell groups is greater than that of the control groups.

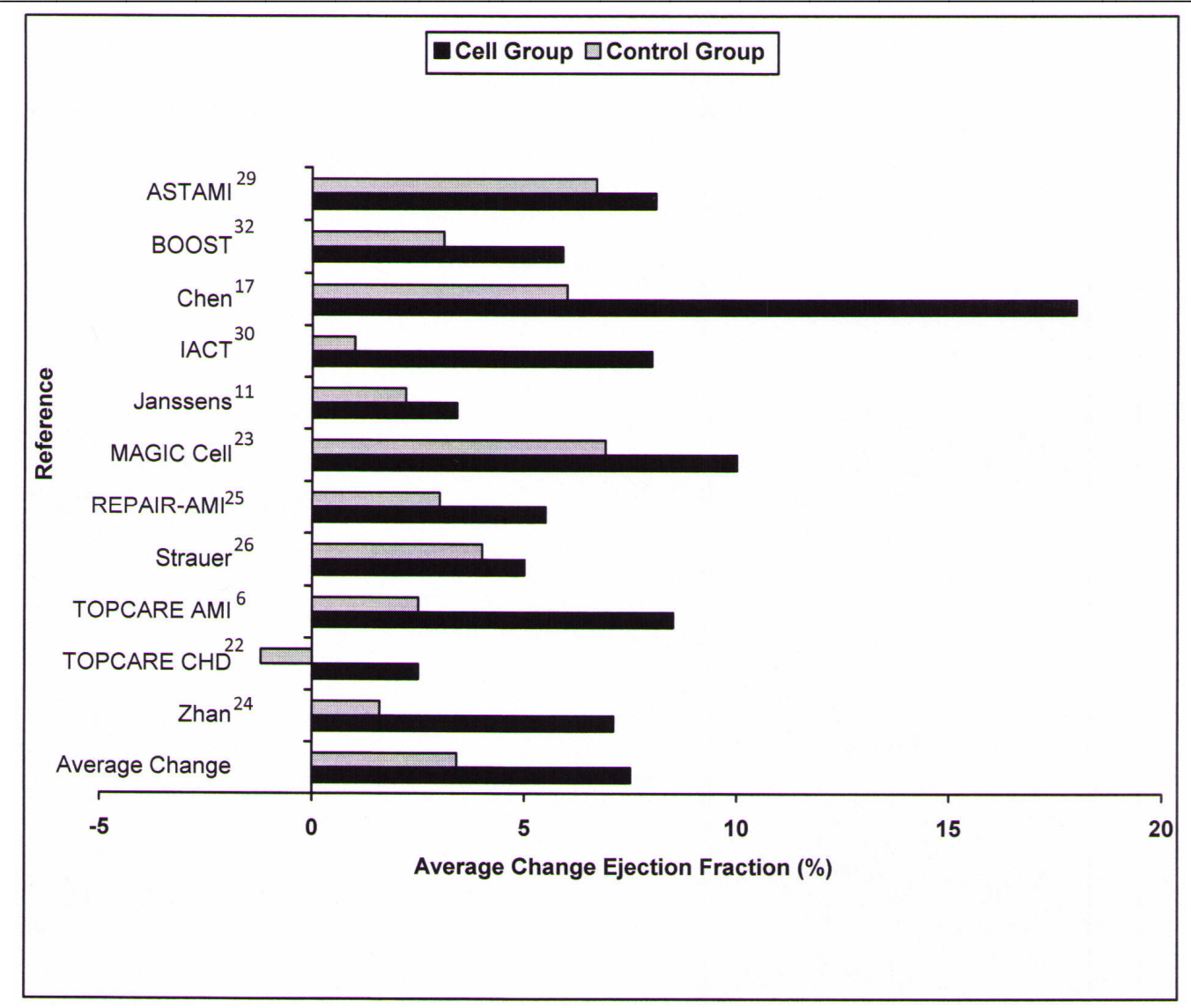

Figure 1. Average Change in Ejection Fraction of Clinical Trials 
In a few studies, the improvement is only slightly more than that of the control; however, there is always a greater increase in the cell therapy group. The compiled data in Table 2 indicates average changes in ejection fraction of 11 clinical studies. Change in LVEF in the cell treatment groups range from $+5 \%$ to $+18 \%$, whereas the control groups had a range from $-1.2 \%$ to only $+7.9 \%$. The average improvement in ejection fraction calculated in the cell therapy group compared to the control groups was +4.1 $(55 \%)$ with a range of $+0.2(3 \%)$ to $+12(88 \%)$, indicating significant increases in cardiac function. The cell infusion groups of acute MI trials had average decreases in left ventricular EDV and ESV after follow-up analyses, and the control groups had average increases in both EDV and ESV, as shown in Table 3. The average decrease in EDV and ESV correlates with a decrease in the size of the left ventricle, indicating the possible prevention of left ventricular dilation that often transpires post-MI.

Table 2. Effects of Stem Cell Therapy in Acute and Chronic Ml on Ejection Fraction ${ }^{\mathrm{a}}$

\begin{tabular}{|c|c|c|c|c|c|c|}
\hline \multirow{2}{*}{$\begin{array}{l}\text { Clinical Study } \\
\text { Reference }\end{array}$} & \multirow{2}{*}{$\begin{array}{c}\text { Acute/ } \\
\text { Chronic MI }\end{array}$} & \multicolumn{2}{|c|}{ Patients } & \multicolumn{2}{|c|}{ LVEF Change $^{\mathrm{b}}(\%)$} & \multirow{2}{*}{$\begin{array}{c}\text { Treatment Effect on } \\
\text { LVEF } \\
\text { (\% Improvement)c }\end{array}$} \\
\hline & & Cell group & Control & Cell Group & Control & \\
\hline ASTAMI 29 & Acute & $n=50$ & $n=50$ & +8.1 & +7.9 & $+0.2(3 \%)$ \\
\hline BOOST 32 & Acute & $n=30$ & $n=30$ & +5.9 & +3.1 & $+2.8(48 \%)$ \\
\hline Chen ${ }^{17}$ & Acute & $n=34$ & $n=35$ & +18 & +6 & $+12(67 \%)$ \\
\hline IACT Study ${ }^{30}$ & Chronic & $n=18$ & $n=19$ & +8 & +1 & $+7(88 \%)$ \\
\hline Janssens ${ }^{11}$ & Acute & $n=30$ & $n=30$ & +3.4 & +2.2 & $+1.2(35 \%)$ \\
\hline MAGIC Cell ${ }^{23}$ & Mixed & $n=10$ & $n=10$ & +10 & +6.9 & $+3.1(31 \%)$ \\
\hline REPAIR-AMI ${ }^{25}$ & Acute & $n=95$ & $n=92$ & +5.5 & +3 & $+2.5(46 \%)$ \\
\hline Strauer 26 & Acute & $n=10$ & $n=10$ & +5 & +4 & $+1(33 \%)$ \\
\hline TOPCARE AMI ${ }^{6}$ & Acute & $n=19$ & $n=10$ & +8.5 & +2.5 & $+6(71 \%)$ \\
\hline TOPCARE CHD ${ }^{22}$ & Chronic & $n=69$ & $n=23$ & +2.5 & -1.2 & $+3.7(76 \%)$ \\
\hline Zhan 24 & Acute & $n=35$ & $n=23$ & +7.1 & +1.6 & $+5.5(78 \%)$ \\
\hline Averages & & & & +7.5 & +3.4 & $+4.1(55 \%)$ \\
\hline
\end{tabular}

In clinical studies that investigated the effects of stem cell therapy on the region of infarction, there is evidence of significant decreases in infarct size and ventricular contractility. During an active Ml, the immediate perimeter around the dead tissue is surrounded by a region of ischemia. Once there is reperfusion to the coronary artery, the ischemia can be resolved but the infarct zone remains. The infarct zone contains non-functional myocytes that are normally remodeled by scar tissues. With current standard treatment, the infarct zone has little to no recovery in function; however, adult stem cells have the ability to considerably reduce the extent of infarction. Table 4 provides compiled data on the effects of stem cell therapy of infarct size, providing evidence of changes up to $60 \%$. The ICAT and TOPCARE-CHD studies were clinical trials of patients with chronic Ml, where recovery using current therapy was near the maximum. ${ }^{22,30}$ Revival of the cardiac tissue in the infarct zone can be attributed to improvement in the contractile function of the left ventricle and enhances the functional activity of the heart. ${ }^{28}$ The remodeling of the tissue within the infarct region is imperative to the increased function of the heart, and it is physiologically improbable that the typical scar tissue is responsible for such a significant improvement. The ICAT study was also able to show a $15 \%$ increase in glucose uptake in the recovered infarct region, indicating a regeneration of myocardial tissues capable of normal function. The improvement of the myocardium in the infarct border zone has resulted in beneficial increases in wall motion and systolic function. ${ }^{32}$ Additional studies have also indicated comparable results of contractile recovery, demonstrating that adult stem cell therapy is effective in providing functional recovery of myocardium. ${ }^{25,26}$

In a number of $\mathrm{Ml}$ cases, the existing coronary vascular supply is compromised by plaque formations that lead to the arterial blockage. Since the existing vasculature is not sufficient to supply the myocardial tissue with the critically needed oxygen and nutrients, new blood vessel formation, or angiogenesis, would likely benefit the infarct regions after an Ml. Stem cell transplantation has shown to induce angiogenesis at the site of injury. 
Table 3. Comparison of Volume Changes in Acute MI Clinical Trials ${ }^{\mathrm{a}}$

\begin{tabular}{|c|c|c|c|c|c|}
\hline \multirow[t]{2}{*}{ Clinical Study Reference } & \multirow[t]{2}{*}{ Patients } & \multicolumn{2}{|c|}{ LV EDV (ml) } & \multicolumn{2}{|c|}{ LV ESV (ml) } \\
\hline & & Baseline & Follow-up & Baseline & Follow-up \\
\hline \multicolumn{6}{|l|}{ ASTAMI ${ }^{29}$} \\
\hline BMSC Infusion & $n=50$ & $162.3 \pm 59$ & $151.1 \pm 52$ & $\mathrm{n} / \mathrm{a}$ & $\mathrm{n} / \mathrm{a}$ \\
\hline Control & $n=50$ & $148.0 \pm 46$ & $146.0 \pm 50$ & $\mathrm{n} / \mathrm{a}$ & $\mathrm{n} / \mathrm{a}$ \\
\hline \multicolumn{6}{|l|}{ Chen ${ }^{17}$} \\
\hline BMSC Infusion & $n=34$ & $169.0 \pm 21$ & $131.0 \pm 19$ & $76.0 \pm 18$ & $58.0 \pm 13$ \\
\hline Control & $n=35$ & $\mathrm{n} / \mathrm{a}$ & $\mathrm{n} / \mathrm{a}$ & $\mathrm{n} / \mathrm{a}$ & $\mathrm{n} / \mathrm{a}$ \\
\hline \multicolumn{6}{|l|}{ MAGIC Cell ${ }^{23}$} \\
\hline CPC Infusion & $n=10$ & $133.0 \pm 34$ & $111.7 \pm 37$ & $70.3 \pm 28$ & $61.8 \pm 35.8$ \\
\hline Control & $n=10$ & $124.2 \pm 33$ & $134.6 \pm 50$ & $61.8 \pm 35$ & $67.9 \pm 44$ \\
\hline \multicolumn{6}{|l|}{ REPAIR-AMI 25} \\
\hline BMSC Infusion & $n=95$ & $128 \pm 38$ & $141 \pm 43$ & $67 \pm 26$ & $67 \pm 30$ \\
\hline Control & $n=92$ & $139 \pm 46$ & $153 \pm 57$ & $75 \pm 32$ & $80 \pm 45$ \\
\hline \multicolumn{6}{|l|}{ TOPCARE AMI ${ }^{6}$} \\
\hline BMSC/CPC Infusion & $n=19$ & $117.2 \pm 35$ & $105.2 \pm 29$ & $56.1 \pm 20$ & $42.2 \pm 15$ \\
\hline Control & $n=10$ & $102.0 \pm 23$ & $123.0 \pm 50$ & $50.4 \pm 17$ & $58.2 \pm 32$ \\
\hline \multicolumn{6}{|l|}{ Zhan 24} \\
\hline CPC Infusion & $n=35$ & $134.2 \pm 36$ & $119.2 \pm 30$ & $63.8 \pm 24$ & $52.6 \pm 20$ \\
\hline Control & $n=23$ & $122.1 \pm 35$ & $113.5 \pm 24$ & $59.4 \pm 25$ & $52.6 \pm 9$ \\
\hline \multicolumn{6}{|l|}{ Averages } \\
\hline Cell infusion & & 140.6 & 126.5 & 66.6 & 56.3 \\
\hline Control & & 127.0 & 134.0 & 61.6 & 64.6 \\
\hline
\end{tabular}

Animal research first discovered this increase in capillary density which allowed an increase in the number of stem cells that reached the damaged tissue, permitting a greater amount of regeneration. It was also suggested that the neovascularization allowed for a more effective delivery of the necessary nutrients and growth factors to allow for greater proliferation of the new cells. ${ }^{33}$ In clinical studies, there is evidence that the capillary and larger vessel density was greater in the infarct region of stem cell treated patients than in the control patients. It was also noted that the beneficial effects from the neovascularization and myocardial regeneration was maintained for 4 years in the stem cell patient groups. ${ }^{31}$ The increased perfusion is imperative to the functional recovery and can be attributed to improvements of cardiac performance at rest and during stress. ${ }^{23}$ The stimulation of angiogenesis is reassuring and indicated that stem cell therapy has a number of positive effects in treatment of Ml. The vessel formation in initial treatment could be beneficial in future analysis of repeat treatments in stem-cell-treated patients since the revascularization provides greater opportunity for the cells to reach infarct regions. 
Table 4. Change in Infarct Size for Stem Cell Therapy Trials in Mla

\begin{tabular}{|c|c|c|c|c|}
\hline \multirow[t]{2}{*}{ Clinical Study } & \multirow[t]{2}{*}{ Patients (n) } & \multicolumn{3}{|c|}{ Change in Extent of Infarction (\%) } \\
\hline & & Pre-Treatment & Post-Treatment & Change- no. (\%) \\
\hline \multicolumn{5}{|l|}{ IACT Study ${ }^{30}$} \\
\hline BMSC Infusion & $n=18$ & 27 & 19 & $-6(30 \%)$ \\
\hline Control & $n=18$ & 27 & 26 & $-1(4 \%)$ \\
\hline \multicolumn{5}{|l|}{ Strauer ${ }^{26}$} \\
\hline BMSC Infusion & $n=10$ & 30 & 12 & $-18(60 \%)$ \\
\hline Control & $n=10$ & 25 & 20 & $-5(20 \%)$ \\
\hline \multicolumn{5}{|l|}{ TOPCARE CHD ${ }^{22}$} \\
\hline BMSC Infusion & $n=35$ & 45 & 42 & $-3(7 \%)$ \\
\hline CPC Infusion & $n=34$ & 52 & 50 & $-2(4 \%)$ \\
\hline Control & $n=23$ & 45 & 45 & $0(0 \%)$ \\
\hline
\end{tabular}

The recovery acquired from the utilization of adult stem cells has been observed to have sustained clinical benefit for up to 4 years after therapy. Several studies have corroborating data indicating that the functional recovery is not temporary, but remains a long term advantage to patients with myocardial damage.23,28,31 The follow-up data on cell treatment patients demonstrates that the myocardial and functional recovery continuously increased through the entire treatment period, indicating that stem cells have a continuous influence on remodeling, even after only one regimen of stem cells. ${ }^{23}$ With functional benefit after a single dose, adult stem cells hold a greater long-term clinical benefit than current medical treatment alone.

When chronic and acute Ml clinical study data was compared, it was found that there is no significant difference in myocardial benefit. The TOPCARE CHD and ICAT studies focused on chronic MI patients and the MAGIC CELL study combined both chronic and acute. 22,23,30 In evaluation of the LVEF data compiled in Table 2, there is no significant difference between the chronic Ml studies and the remaining acute Ml studies. However, the stem cell treatment effects showed improvements of $76 \%$, $88 \%$, and $31 \%$ respectively, compared to the control patients. The control patients in the chronic Ml studies did not have a significant functional recovery on standard therapies, and in the ICAT report, there was a negative average LVEF change. ${ }^{30}$ Considering chronically infarcted myocardium typically reaches a peak in functional recovery on standard therapy, the chronic treatment is focused on maintenance. With stem cell treatment, the evidence demonstrates that functional recovery is attainable after the standard therapy has reached a peak in the remodeling process. Therefore, those patients that do not elect stem cell treatment in the acute setting will still gain functional recovery from treatment months to years post-MI.

The majority of studies have focused on patients with only mild loss in cardiac function and a LVEF typically not less than $45 \%$. In several studies, there has been substantial documented evidence that patients with a worse baseline cardiac function had a larger overall recovery with cell therapy and significantly greater recovery than the control patients.11,23,25 The areas of most severe infarction gained the most benefit from cell therapy. In other words, the patients with more room to recover encountered a wider range of improvement from the stem cell therapy. In response to findings of impairment-dependant recovery, it was reported that "when combined with optimal reperfusion therapy (stent implantation) and state-of-the-art medical treatment, intracoronary administration of [BMSC] enhances the recovery of global and regional left ventricular function after myocardial infarction."25(p1220) In clinical research of adult stem cell treatment of MI, there are a few issues that still remain: 1) the lack of large population testing in one study group, 2) the lack of studies involving patients with moderate to severe cardiac impairment, 3 ) the lack of consistency of stem cell collection, and 4) unknown optimal cell concentrations. However, these issues are 
relatively trivial to future implementation of adult stem cell therapy, considering the known benefits attained though the data provided from the current clinical studies.

\section{SAFETY AND MONITORING OF STEM CELL THERAPY}

It is important during the implementation of adult stem cell therapy to establish a satisfying safety profile. Many of the adverse events encountered during clinical studies were related to the $\mathrm{PCl}$ and stent procedures prior to therapy and non-treatment related issues rather than the stem cell therapy. The safety profile of $\mathrm{PCl}$ has already been established, but during clinical trials the major complications included 1) local dissection, 2) ventricular fibrillation, 3) stent thrombosis, and 4) ventricular tachycardia.22,24,29 In studies that utilized G-CSF, there were increased complications as a result of the mobilization of cells into circulation, and patients were at increased risk for thrombosis and bone pain. ${ }^{24}$ In evaluation of stem cell efficacy and safety, follow-up visits were necessary and regular throughout the patient treatment periods. In future treatments, continuous follow-up visits will be necessary for patients to monitor cardiac function and potential side effects.

The collection and infusion procedures of stem cells have demonstrated minimal side effects and have shown to be a safe and feasible treatment for MI. In the 12 clinical studies investigated, all indicate through the reported results that adult stem cell therapy is a safe method of treatment for MI patients. Several reports express a lack of serious adverse reactions to the treatment, specifically illustrating no inflammatory processes, malignant arrhythmias, ischemic damages from infusion, or deaths. ${ }^{6,23,26,30}$ Complications that have transpired include restenosis, reinfarction, and microbial contamination. ${ }^{29,32}$ Many complications that arose during the treatment period were no more prevalent in stem cell treated patients than in the control patients receiving standard therapy. The stem cell therapy has shown to actually reduce recurrence of Ml, which is a typical chronic effect of ischemia and coronary stenosis. ${ }^{25}$ In addition, treatment has not shown any significant difference between male and female populations. ${ }^{6}$ This evidence gives strong support to the safety profile of stem cell administration.

\section{DISCUSSION}

Clinical studies have provided sufficient evidence that adult stem cells are a viable resource in the treatment of acute and chronic myocardial infarctions. Stem cells can be obtained from a variety of locations, but the most beneficial outcome is obtained from bone marrow stem cells with delivery via over-the-wire balloon catheterization. Important changes that have been documented after treatments with adult stem cells include 1) formation of new cardiomyocytes, 2) sufficient and sustained improvements in cardiac output, 3) increased myocardial contractility, 4) decreased infarct zone diameter, 5) increased left ventricular function, 6) increased exercise ability, and 7) increased coronary perfusion secondary to neovascularization. The improvement in cardiac function aids to reduce the detrimental effects of chronic $\mathrm{Ml}$ and prevents further complications in the patient. The functional benefits of adult stem cells are attained after a single dose and carry greater long-term clinical benefits than the current standard medical treatment. In addition, stem cell therapy has shown to reduce the rate of recurrence of myocardial infarction.

At this time, based on the current clinical evidence, adult stem cell therapy is in a position to be considered as an optional treatment for patients with acute or chronic Ml. Although stem cell therapies are still considered experimental, the continued clinical involvement of adult stem cell therapy will provide more evidence to the therapeutic effects of the treatment. At the moment, consideration needs to be given to those patients with severe cardiac functional deficits where current medical treatments are at a maximal effect. This is further supported by the evidence that stem cell therapy has more favorable effect on those patients with greater functional deficits. Patients with chronically sustained low cardiac output and ejection fractions less than $25 \%$ are at higher risk for further myocardial as well as systemic complications and need new additional treatments to increase cardiac survival. The benefits of stem cell therapy in such patients outweigh the minimal risks associated with the treatment. Patients with relative decreases in cardiac output and ejection fractions between $25 \%$ and $55 \%$ will still benefit from stem cell administration, and treatment options need to be made available to these patients. The prospect for adult stem cell therapy is promising for the future management myocardial tissue damage. With an excellent safety profile and the ease of onetime dosing, adult stem cell therapy has shown dramatic functional improvements and reduces the recurrence of MI while enhancing quality of life for myocardial infarction patients.

\section{REFERENCES}

1. Kung HC, Hoyert DL, Xu J, Murphy SL. Deaths: Final data for 2005. Natl Vital Stat Rep[Online]. January 2008;56(10):1-66. http://www.cdc.gov/nchs/data/nvsr/nvsr56/nvsr56_10.pdf. Accessed February 17, 2008.

2. Hellerman JP, Jacobsen SJ, Gersh BJ, Rodeheffer RJ, Reeder GS, Roger VL. Heart failure after myocardial infarction: A review. Am J Med. 2002:113:324-330.

3. Sutton MG, Sharpe N. Left ventricular remodeling after myocardial infarction: Pathophysiology and therapy. Circulation. 2000;101:2981-2988. 
4. Davani S, Marandin A, Mersin N, et al. Mesenchymal progenitor cells differentiate into an endothelial phenotype, enhance vascular density, and improve heart function in a rat cellular cardiomyoplasty model. Circulation. 2003; 108[suppl II]:II253II258.

5. Dawn B, Stein AB, Urbanek K, et al. Cardiac stem cells delivered intravascularly traverse the vessel barrier, regenerate infarcted myocardium, and improve cardiac function. Proc. Natl. Acad. Sci. 2005; 102:10344-10349.

6. Assmus B, Schächinger V, Teupe C, et al. Transplantation of progenitor cells and regeneration enhancement in acute myocardial infarction (TOPCARE-AMI). Circulation. 2002; 106:3009-3017.

7. Jain M, DerSimonian $\mathrm{H}$, Brenner DA, et al. Cell therapy attenuates deleterious ventricular remodeling and improves cardiac performance after myocardial infarction. Circulation. 2001; 103:1920-1927.

8. Thygesen K, Alpert JS, White HD, et al. Universal definition of myocardial infarction. Circulation. 2007;116:2634-2653.

9. Smith SC, Jacobs AK, Adams CD, et al. 2007 focused update of the ACC/AHA 2004 guidelines for the management of patients with ST-elevation myocardial infarction. Circulation. 2008;117:296-329.

10. Anderon JL, Adams CD, Antman EM, et al. ACC/AHA 2007 guidelines for the management of patients with unstable angina/non-ST-elevation myocardial infarction. Circulation. 2007;116:e148-e304.

11. Janssens S, Dubois C, Bogaert J, et al. Autologous bone marrow-derived stem-cell transfer in patients with ST-segment elevation myocardial infarction: double blind, randomized controlled trial. Lancet. 2006;367: 113-121.

12. Makino S, Fukuda K, Miyoshi S, et al. Cardiomyocytes can be generated from bone marrow stromal cells in vitro. J. Clin. Invest. 1999; 103:697-705.

13. Matsuura K, Nagai T, Nishigaki N, et al. Adult cardiac sac-1-positive cells differentiate into beating cardiomyocytes. J. Biol. Chem. 2004; 279: 11384-11391.

14. Urbanek K, Quaini F, Tasca G, et al. Intense myocyte formation from cardiac stem cells in human cardiac hypertrophy. Proc. Natl. Acad. Sci. 2003; 100: 10440-10445.

15. Müller $P$, Pfieffer $P$, Koglin J, et al. Cardiomyocytes of noncardiac origin in myocardial biopsies of human transplanted hearts. Circulation. 2002; 106:31-35.

16. Liu J, Hu Q, Wang Z, Xu C, Wang X, Gong G, et al. Autologous stem cell transplantation for myocardial repair. Am. J. Physiol. Heart Circ. Physiol. 2004; 287:H501-H511.

17. Chen S, Fang W, Ye F, et al. Effect on left ventricular function of intracoronary transplantation of autologous bone marrow mesenchymal stem cell in patients with acute myocardial infarction. Am. J. Cardiol. 2004; 94:92-95.

18. Fuchs S, Satler LF, Kornowski R, et al. Catheter-based autologous bone marrow myocardial injection in no-option patients with advanced coronary artery disease. Am J Cardiol. 2003;41:1721-1724.

19. Kuramochi $Y$, Fukazawa R, Migita M, et al. Cardiomyocyte regeneration fom circulating bone marrow cells in mice. Pediatric Research. 2003; 54:319-325.

20. Orlic D, Kajstura J, Chimenti S, et al. Bone marrow cells regenerate infracted myocardium. Nature. 2001; 410:701-705.

21. Toma C, Pittenger MF, Cahill KS, Byrne BJ, Kessler PD. Human mesenchymal stem cells differentiate to a cardiomyocyte phenotype in the adult murine heart. Circulation. 2002; 105:93-98.

22. Assmus $B$, Honold J, Schächinger $V$, et al. Transcoronary transplantation of progenitor cells after myocardial infarction. $N$ Engl J Med. 2006;355:1222-1232.

23. Kang HJ, Kim HS, Koo BK, et al. Intracoronary infusion of the mobilized peripheral blood stem cell by G-CSF is better than mobilization alone by G-CSF for improvement of cardiac function and remodeling: 2-year follow-up results of the myocardial regeneration and angiogenesis in myocardial infarction with G-CSF and intracoronary stem cell infusion (MAGIC Cell) 1 trial. Am Heart J. 2007;153:237e1-237e8.

24. Zhan-quan L, Ming Z, Yuan-zhe J, Wei-wei Z, Ying L, Li-jie C, et al. The clinical study of autologous peripheral blood stem cell transplantation by intracoronary infusion in patients with acute myocardial infarction (AMI). Int J Cardiol. 2007;115:5256.

25. Schächinger V, Erbs S, Elsässer A, Haberbosch W, Hambrecht R, Hölschermann H, et al. Intracoronary bone marrowderived progenitor cells in acute myocardial infarction. N Engl J Med. 2006;355:1210-1221.

26. Strauer BE, Brehm M, Zeus T, Köstering M, Hernandez A, Sorg RV, Kögler G, Wernet P. Remair of infarcted myocardium by autologous intracoronary mononuclear bone marrow cell transplantation in humans. Circulation. 2002; 106:1913-1918.

27. Nagaya N, Fujii T, Iwase T, et al. Intravenous administration of mesenchymal stem cells improves cardiac function in rats with acute myocardial infarction through angiogenesis and myogenesis. Am. J. Physiol. Heart Circ. Physiol. 2004; 287:H2670-H2675.

28. Britten MB, Abolmaali ND, Assmus B, Lehmann R, Honold J, Schmitt J, et al. Infarct remodeling after intracoronary progenitor cell treatment for patients with acute myocardial infarction (TOPCARE-AMI): Mechanistic insights from serial contrast-enhanced magnetic resonance imaging. Circulation. 2003; 108:2212-2218.

29. Lunde K, Solheim S, Aakhus S, Arnesen H, Abdelnoor M, Egeland T, et al. Intracoronary injection of mononuclear bone marrow cells in acute myocardial indarction. N Engl J Med. 2006;355:1199-1209. 
30. Strauer BE, Brehm M, Zeus T, Bartsch T, Schannwell, Antke C, Sorg R, Kögler G, Wernet P, Müller H, Köstering M. Regeneration of human infarcted heart muscle by intracoronary autologous bone marrow cell transplantation in chronic coronary artery disease. J Am Coll Cardiol. 2005;46:1651-8.

31. Dohmann HFR, Silva SA, Souza ALS, Rosi MI, Takiya CM, Borojevic R. Bone-marrow mononuclear cell therapy of severe ischemic heart failure. C.R. Biologies. 2007; 330: 543-549.

32. Wollert KC, Lotz J, Ringes S, Lippolt P, Breidenbach C, Fichtner S, et al. Intracoronary autologous bone-marrow cell transfer after myocardial infarction: the BOOST randomized controlled clinical trial. Lancet. 2004;364:141-148.

33. Schuster MD, Kocher AA, Seki T, Martens TP, Xiang G, Homma S, Itescu S. Myocardial neovascularization by bone 